\title{
SMOOTH APPROXIMATIONS IN BANACH SPACES
}

\author{
J. VANDERWERFF
}

(Communicated by William J. Davis)

\begin{abstract}
A Banach space that has a locally uniformly convex (LUC) norm whose dual is also LUC is shown to admit $C^{1}$-smooth partitions of unity. It is also established that there is a norm on a Hilbert space with Lipschitz derivative that cannot be approximated uniformly on bounded sets by functions with uniformly continuous second derivative.
\end{abstract}

\section{INTRODUCTION}

The study of smooth partitions of unity in Banach spaces is of interest as it provides a tool for approximating continuous functions by smooth functions (see $[1,18])$. While the situation in separable spaces has long been settled [1], in nonseparable spaces, smooth partitions of unity are also important because they are related to injections into $c_{0}(\Gamma)$. The majority of results showing the existence of smooth partitions of unity on nonseparable Banach spaces are obtained by constructing coordinatewise smooth embeddings into some $c_{0}(\Gamma)$ and applying the fundamental result of Torunczyk found in [18]. Usually such embeddings of $X$ into $c_{0}(\Gamma)$ are constructed with the help of a linear injection of $X$ into $c_{0}(\Gamma)$. In this direction, the combined efforts of [8] and [13] show that $X$ admits $C^{k}$-smooth partitions of unity whenever $X$ has a $C^{k}$-smooth bump function and $X$ or $X^{*}$ is WCG. However, $C^{1}$-smooth partitions of unity on $X$ are obtained here by the purely geometric condition that $X$ admits an equivalent LUC norm whose dual is also LUC. Such a space need not linearly inject into any $c_{0}(\Gamma)$; notwithstanding, from [18] and our result stated above, there is a coordinatewise smooth embedding of such a space into some $c_{0}(\Gamma)$.

The $C(K)$ spaces are of particular interest. In [2] Ciesielski and Pol constructed a $C(K)$ space such that $K^{(3)}=\varnothing$ while $C(K)$ does not linearly inject into any $c_{0}(\Gamma)$. Only recently it was shown in [4] that $C(K)$ admits $C^{\infty}$ smooth partitions of unity whenever $K^{\left(\omega_{0}\right)}=\varnothing$. In fact, until [4], it had been unknown whether the $C(K)$ space of [2] admits $C^{1}$-smooth partitions of unity. Using renormings of Deville [3] and Haydon and Rogers [12], it is shown here

Received by the editors September 12, 1990 and, in revised form, November 20, 1990.

1980 Mathematics Subject Classification (1985 Revision). Primary 46B05, 46B20, Secondary $54 \mathrm{C} 35,54 \mathrm{G} 20$.

This paper is based on part of the author's Ph.D. thesis written under the supervision of Professor V. Zizler 
that $C(K)$ admits $C^{1}$-smooth partitions of unity whenever $K^{\left(\omega_{1}\right)}=\varnothing$. In [10] Haydon constructed a $C(K)$ space such that $K^{\left(\omega_{1}\right)}$ is a singleton, which nevertheless admits no equivalent Gâteaux smooth nor strictly convex norm. It is presently unknown whether this fundamentally important space admits $C^{1}$ smooth partitions of unity, although Haydon has recently shown [11] that there is a Fréchet smooth bump function on it.

The notation and terminology used here should be quite standard. A norm is LUC if $\left\|x_{n}-x\right\| \rightarrow 0$ whenever $\left\|x_{n}\right\| \rightarrow\|x\|=1$ and $\left\|x_{n}+x\right\| \rightarrow 2$. Further properties of LUC norms can be found in [5]. As usual, $C^{k}$-smoothness is always in the continuous Fréchet sense. We call a function $C^{k}$-smooth if it is $C^{k}$-smooth on the whole space. A norm on $X$ will be called Fréchet (Gâteaux differentiable) if it is is Fréchet (Gâteaux) differentiable on $X \backslash\{0\}$. A space is said to admit $C^{k}$-smooth partitions of unity if given any open cover there is a locally finite partition of unity consisting of $\widetilde{C}^{k}$-smooth functions subordinate to this cover. The notation $\partial f\left(x_{0}\right)=\left\{\Lambda \in X^{*}: \Lambda(x)-\Lambda\left(x_{0}\right) \leq\right.$ $f(x)-f\left(x_{0}\right)$ for all $\left.x \in X\right\}$, and for $\varepsilon>0, \partial_{\varepsilon} f\left(x_{0}\right)=\left\{\Lambda \in X^{*}: \Lambda(x)-\right.$ $\Lambda\left(x_{0}\right) \leq f(x)-f\left(x_{0}\right)+\varepsilon$ for all $\left.x \in X\right\}$ will be used. We also use the symbols $S_{X}=\{x:\|x\|=1\}, B_{r}\left(x_{0}\right)=\left\{x:\left\|x-x_{0}\right\| \leq r\right\}, B_{r}=B_{r}(0)$, and $B_{x}=B_{1}$.

\section{SMOOTH PARTITIONS OF UNITY}

The main result of this note is

Theorem 2.1. (a) If $X$ has an LUC norm whose dual is also LUC, then $X$ admits $C^{1}$-smooth partitions of unity.

(b) If $X$ has an LUC norm whose dual is strictly convex $(S C)$, then $X$ admits Gâteaux smooth partitions of unity.

Recall that from Asplund's averaging technique (see [5]), it follows that if $X$ has an LUC norm and $X^{*}$ has a dual LUC (respectively SC) norm, then $X$ has an LUC norm whose dual norm is LUC (respectively SC).

Corollary 2.2. (a) If there is a $w^{*}$-compact $K \subset X^{*}$ such that $\left(K, w^{*}\right)^{\left(\omega_{1}\right)}=\varnothing$ and the linear span of $K$ is norm-dense in $X^{*}$, then $X$ admits $C^{1}$-smooth partitions of unity.

(b) If $K^{\left(\omega_{1}\right)}=\varnothing$, then $C(K)$ admits $C^{1}$-smooth partitions of unity.

(c) If $X^{*}$ is weakly countably determined (WCD) (in particular when $X^{*}$ is a subspace of a WCG space $Y$ ), ithen $X$ admits $C^{1}$-smooth partitions of unity. Proof of Corollary 2.2. All three parts follow immediately from known renormings and Theorem 2.1(a). We briefly indicate where such renormings are found. From [3] and [12] and Asplund's averaging technique, it follows that $C(K)$ has an LUC norm whose dual is LUC whenever $K^{\left(\omega_{1}\right)}=\varnothing$. This proves (b). The more general statement (a) follows because the above renorming of $C(K)$ and transfer techniques as used in [3, 8, 9] show that $X$ has an LUC norm whose dual is also LUC. One obtains (c) from the recent papers [6, 7], which show that $X$ has an LUC norm whose dual is LUC whenever $X^{*}$ is WCD. We remark that for $X^{*}$ a subspace of a WCG space the full generality of $[6,7]$ is not needed the results of $[8,9]$ suffice. Also, when $X^{*}$ is WCG, (c) is a special case of (a) with $K^{(2)}=\varnothing$.

The proof of Theorem 2.1 will be separated into several steps. The following 
lemma is a natural generalization of a result of Šmulyan [15]. We include its proof for the reader's convenience.

Lemma 2.3. Let $f$ be a continuous convex function on $X$. Then the following are equivalent:

(a) $f$ is Fréchet differentiable at $x_{0}$.

(b) $\left\|\Lambda_{n}-\Lambda\right\| \rightarrow 0$ whenever $\Lambda \in \partial f\left(x_{0}\right)$ and $\Lambda_{n} \in \partial_{\varepsilon_{n}} f\left(x_{0}\right)$ where $\varepsilon_{n} \downarrow 0$.

Proof. We omit the proof of (a) implies (b) because it is not difficult and it is not used in this note.

(b) implies (a): Suppose $f$ is not Fréchet differentiable at $x_{0}$. Then there exists $t_{n} \downarrow 0, x_{n} \in S_{X}$, and $\varepsilon>0$ such that

$$
f\left(x_{0}+t_{n} x_{n}\right)-f\left(x_{0}\right)-\Lambda\left(t_{n} x_{n}\right) \geq \varepsilon t_{n}, \quad \text { where } \Lambda \in \partial f\left(x_{0}\right) .
$$

Let $\Lambda_{n} \in \partial f\left(x_{0}+t_{n} x_{n}\right)$. Since $f$ is locally Lipschitzian, there exists $\delta>0$ and $M>0$ such that $|f(x)-f(y)| \leq M|| x-y \|$ whenever $x, y \in B_{\delta}\left(x_{0}\right)$. We may assume that $t_{n}<\delta$ for all $n$. Thus $\left\|\Lambda_{n}\right\| \leq M$ for all $n$; moreover,

$$
\begin{aligned}
\Lambda_{n}(y)-\Lambda_{n}\left(x_{0}\right) & =\Lambda_{n}(y)-\Lambda_{n}\left(x_{0}+t_{n} x_{n}\right)+\Lambda_{n}\left(t_{n} x_{n}\right) \\
& \leq f(y)-f\left(x_{0}+t_{n} x_{n}\right)+M t_{n} \leq f(y)-f\left(x_{0}\right)+2 M t_{n} .
\end{aligned}
$$

Whence, for all $n, \Lambda_{n} \in \partial_{\varepsilon_{n}} f\left(x_{0}\right)$, where $\varepsilon_{n}=2 M t_{n} \downarrow 0$. However,

$$
\Lambda_{n}\left(t_{n} x_{n}\right) \geq f\left(x_{0}+t_{n} x_{n}\right)-f\left(x_{0}\right) \geq \Lambda\left(t_{n} x_{n}\right)+\varepsilon t_{n} .
$$

Therefore $\left\|\Lambda-\Lambda_{n}\right\| \geq \varepsilon$ for all $n$. Thus (b) fails.

An easy but useful application of Lemma 2.3 is contained in

Lemma 2.4. Let $C$ be a closed convex subset of $X$, and $\rho(x)=\rho(x, C)=$ $\inf \{\|x-y\|: y \in C\}$.

(a) If $X^{*}$ is LUC, then $\rho(x)$ is Fréchet differentiable at each $x \notin C$.

(b) If $X^{*}$ is $S C$, then $\rho(x)$ is Gâteaux differentiable at each $x \notin C$.

Proof. (a) Let $x_{0} \notin C$ and $\Lambda_{n} \in \partial_{\varepsilon_{n}} \rho\left(x_{0}\right)$ for each $n$, where $\varepsilon_{n} \downarrow 0$. Since $|\rho(x)-\rho(y)| \leq\|x-y\|$, it follows that $\left\|\Lambda_{n}\right\| \leq 1$. Thus $\overline{\lim }|| \Lambda_{n} \| \leq 1$. On the other hand, choosing $x_{n} \in C$ such that $\left\|x_{0}-x_{n}\right\| \rightarrow \rho\left(x_{0}\right)>0$, one has

$$
\frac{\Lambda_{n}\left(x_{0}-x_{n}\right)}{\left\|x_{0}-x_{n}\right\|} \geq \frac{\rho\left(x_{0}\right)-\rho\left(x_{n}\right)-\varepsilon_{n}}{\left\|x_{0}-x_{n}\right\|} \rightarrow 1
$$

Therefore, $\underline{\lim }\left\|\Lambda_{n}\right\| \geq 1$. In particular, $\|\Lambda\|=1$ whenever $\Lambda \in \partial \rho\left(x_{0}\right)$. Also, $\left(\Lambda+\Lambda_{n}\right) / 2 \in \partial_{\varepsilon_{n}} \rho\left(x_{0}\right)$, for $\Lambda, \Lambda_{n}$, and $\varepsilon_{n}$ as chosen above. Now $\left\|\Lambda_{n}\right\| \rightarrow$ $\|\Lambda\|=1,\left\|\left(\Lambda+\Lambda_{n}\right) / 2\right\| \rightarrow 1$ and thus $\left\|\Lambda-\Lambda_{n}\right\| \rightarrow 0$ because $X^{*}$ is LUC. By Lemma 2.3, $\rho$ is Fréchet differentiable at $x_{0}$.

(b) Let $x_{0} \notin C$ and $\Lambda_{1}, \Lambda_{2} \in \partial \rho\left(x_{0}\right)$. From the proof of (a) it follows that $\left\|\Lambda_{1}\right\|=\left\|\Lambda_{2}\right\|=\left\|\left(\Lambda_{1}+\Lambda_{2}\right) / 2\right\|=1$. Since the dual norm on $X^{*}$ is SC, $\Lambda_{1}=\Lambda_{2}$. Therefore $\rho$ is Gâteaux differentiable at $x_{0}$.

Remark 2.5. (a) Observe that $\rho(x, C)$ need not be Gâteaux differentiable if the norm on $X$ is Fréchet differentiable. This follows, for example, because $X=C\left[0, \omega_{1}\right]$ admits a Fréchet differentiable norm while $X^{*}$ has no dual SC norm (see [17]). Thus given any norm on $X, X^{*}$ has a two-dimensional 
subspace $H$ on which its dual norm is not SC. Let $L$ be a closed subspace of $X$ such that $(X / L)^{*}=H$. On finite-dimensional subspaces, a norm is Gâteaux if and only if its dual norm is SC. Therefore, given any norm on $X$, there is a two-dimensional quotient space $X / L$ such that the quotient norm of $X / L$ is not Gâteaux.

(b) For any nonempty set $F, \rho^{2}(x, F)$ is Fréchet differentiable at each point of $F$. In particular, $\rho^{2}(x, C)$ is $C^{1}$-smooth whenever $C$ is a closed and convex set and $X^{*}$ is LUC (because Fréchet differentiable convex functions are $C^{1}$-smooth).

(c) Choosing $C=\{0\}$, we have the well-known fact that the norm on $X$ is Fréchet differentiable if its dual norm is LUC. This fact is sometimes tacitly used in this note. [14].

The next lemma uses ideas from Theorem 3.2 of [1] and Theorem 1.1 of

Lemma 2.6. Suppose $X$ has an LUC norm whose dual is also LUC. Let $f$ be a bounded continuous function on $B_{X}$. Then given $\varepsilon>0$, there exists a $C^{1}$ smooth function $g$ such that $|g(x)-f(x)|<\varepsilon$ for all $x \in S_{X}$.

Proof. One may assume $-1 \leq f(x) \leq 1$ for $x \in B_{X}$. Choose $N$ such that $7 / 2 N<\varepsilon$. Let

$$
\Delta_{i}=\left[\frac{i-1-N}{N}, \frac{i-N}{N}\right] \text { for } i=1, \ldots, 2 N .
$$

Let $Q_{0}=Q_{2 N+1}=\varnothing$, and for $i=1, \ldots, 2 N$ define

$$
Q_{i}=f^{-1}\left(\Delta_{i}\right) \quad \text { and } \quad \widetilde{Q}_{i}=\overline{\operatorname{conv}}\left(B_{X} \backslash\left(Q_{i-1} \cup Q_{i} \cup Q_{i+1}\right)\right) .
$$

For each $i$, it is assumed that $\widetilde{Q}_{i} \neq \varnothing$, since in case $\widetilde{Q}_{i}=\varnothing, g(x)=(i-1) / N$ would work. Now let $r_{i}(x)=\rho^{2}\left(x, \widetilde{Q}_{i}\right)$ for $i=1, \ldots, 2 N$. By Remark 2.5(b), $r_{i}$ is a $C^{1}$-smooth function for each $i$. Let $x_{0} \in S_{X}$ be fixed. Certainly $x_{0} \in Q_{i_{0}}$ for some $i_{0}$. For some $\delta>0$ one has $\left|f\left(x_{0}\right)-f(y)\right|<1 / N$ for all $y \in B_{X}$ such that $\left\|x_{0}-y\right\|<\delta$. It follows that $y \notin B_{X} \backslash\left(Q_{i_{0}-1} \cup Q_{i_{0}} \cup Q_{i_{0}+1}\right)$ whenever $\left\|x_{0}-y\right\|<\delta$. Since $X$ is LUC, there exists $\Lambda \in S_{X^{*}}$ with $\Lambda\left(x_{0}\right)=1$ such that $\left\|y-x_{0}\right\|<\delta$ whenever $y \in B_{X}$ and $\Lambda(y) \geq 1-\alpha$ for some $\alpha>0$. Hence $\overline{\operatorname{conv}}\left(B_{X} \backslash\left(Q_{i_{0}-1} \cup Q_{i_{0}} \cup Q_{i_{0}+1}\right)\right) \subset\{y: \Lambda(y) \leq 1-\alpha\}$. Therefore $r_{i_{0}}\left(x_{0}\right) \geq \alpha^{2}>0$. Since $x_{0}$ was arbitrary, this shows that $\sum_{i=1}^{2 N} r_{i}(x)>0$ for each $x \in S_{X}$. Let $r(x)=\left(1-\|x\|^{2}\right)^{2}$. Notice that $r(x)=0$ for all $x \in S_{X}, r(x)>0$ for $x \notin S_{X}$, and $r$ is $C^{1}$-smooth. Therefore,

$$
h_{i}(x)=\frac{r(x)+r_{i}(x)}{r(x)+\sum_{i=1}^{2 N} r_{i}(x)}
$$

is $C^{1}$-smooth. Let $\alpha_{i}=$ midpoint of $\Delta_{i}$ and

$$
g(x)=\sum_{i=1}^{2 N} \alpha_{i} h_{i}(x)
$$

Certainly $g$ is $C^{1}$-smooth. Finally for $x_{0} \in S_{X}$ choose $i_{0}$ such that $x_{0} \in Q_{i_{0}}$ 
and use the fact that $r_{i}\left(x_{0}\right)=0$ for $i \notin\left\{i_{0}-1, i_{0}, i_{0}+1\right\}$ to estimate

$$
\begin{aligned}
\left|g\left(x_{0}\right)-f\left(x_{0}\right)\right| & =\left|\frac{\sum_{i=1}^{2 N} \alpha_{i} r_{i}\left(x_{0}\right)}{\sum_{i=1}^{2 N} r_{i}\left(x_{0}\right)}-f\left(x_{0}\right) \frac{\sum_{i=1}^{2 N} r_{i}\left(x_{0}\right)}{\sum_{i=1}^{2 N} r_{i}\left(x_{0}\right)}\right| \\
& \leq \sum_{i=1}^{2 N}\left|\alpha_{i}-f\left(x_{0}\right)\right| \frac{r_{i}\left(x_{0}\right)}{\sum_{i=1}^{2 N} r_{i}\left(x_{0}\right)} \\
& \leq\left|\alpha_{i_{0}-1}-f\left(x_{0}\right)\right|+\left|\alpha_{i_{0}}-f\left(x_{0}\right)\right|+\left|\alpha_{i_{0}+1}-f\left(x_{0}\right)\right| \\
& \leq \frac{7}{2 N}<\varepsilon .
\end{aligned}
$$

Proposition 2.7. Suppose $X$ has an LUC norm whose dual is also LUC, then every bounded continuous function can be approximated uniformly on bounded sets by $C^{1}$-smooth functions.

Proof. Consider $Y=X \oplus \mathbb{R}$ with norm $\|(x, r)\|_{Y}=\left(\|x\|^{2}+|r|^{2}\right)^{1 / 2}$. Certainly $\|\cdot\|_{Y}$ and its dual norm are LUC. Let $x_{0}=(0,1) \in S_{Y}$. Now $\Lambda \in S_{Y^{*}}$ defined by $\Lambda(x, r)=r$ is the supporting functional at $x_{0}$ and $H=\{(x, r): \Lambda(x, r)=$ $1\}=\{(x, 1): x \in X\}$ is the supporting hyperplane. Let $f$ be a bounded continuous function on $H$ and $C$ be a bounded subset of $H$. Thus for some $m\|y\|_{Y}>0, C \subset\{(x, 1):\|x\| \leq m\} \equiv F$. Define $p: Y \backslash\{0\} \rightarrow S_{Y}$ by $p(y)=$ $y /\|y\| Y$; let $p_{1}=\left.p\right|_{H}$. For $p_{1}^{-1}(y) \in F$, set $f_{1}(y)=f\left(p_{1}^{-1}(y)\right)$. Extend $f_{1}$ to a bounded continuous function on $Y$ and denote it again by $f_{1}$. By Lemma 2.6 choose a $C^{1}$-smooth function $g_{1}$ such that $\left|g_{1}(y)-f_{1}(y)\right|<\varepsilon$ for an arbitrary fixed $\varepsilon>0$ and all $y \in S_{Y}$. For $y \in Y$ define $g(y)=g_{1}(p(y))$. Because $\|\cdot\|$ is Fréchet differentiable, $g$ is $C^{1}$-smooth on $Y \backslash\{0\}$. In particular, $g$ is $C^{1}$-smooth on $H$. For $h \in F$, one computes

$$
|g(h)-f(h)|=\left|g_{1}(p(h))-f\left(p_{1}^{-1}(p(h))\right)\right|=\left|g_{1}(p(h))-f_{1}(p(h))\right|<\varepsilon .
$$

Since $H$ is a translate of $X$, the proposition is proved.

Proof of Theorem 2.1. (a) Let $S$ be the set of real-valued $C^{1}$-smooth functions on $X$ and $\mathscr{U}_{S}$ be the family of sets $\left\{f^{-1}(0, \infty): f \in S\right.$ and $\left.f: X \rightarrow[0,1]\right\}$. By Lemma 1 of [18] it suffices to show that $\mathscr{U}_{S}$ contains a $\sigma$-locally finite base for the norm topology on $X$. To accomplish this, we use a technique from Lemma 6 of [16]. Let $O$ be a bounded open subset of $X$, and let $F=X \backslash O$. Choose $r>0$ such that $O \subset B_{r}$. Let $\theta: \mathbb{R} \rightarrow[0,1]$ be a $C^{1}$-smooth function such that $\theta(t)=1$ if $t \leq r^{2}$ and $\theta(t)=0$ if $t \geq(r+1)^{2}$. Since $\|\cdot\|^{2}$ is $C^{1}$-smooth, $\phi(x)=\theta\left(\|x\|^{2}\right)$ is a $C^{1}$-smooth function. Let $\phi_{n}: \mathbb{R} \rightarrow[0,1]$ be a $C^{1}$-smooth function such that

$$
\phi_{n}(t)= \begin{cases}0 & \text { if } t \leq 1 / 2 n \\ >0 & \text { otherwise }\end{cases}
$$

By Proposition 2.7, one can choose a $C^{1}$-smooth function $h_{n}$ such that $\mid h_{n}(x)-$ $\rho(x, F) \mid<1 / 2 n$ for $x \in B_{r+1}$. Set $g_{n}(x)=\phi(x) \phi_{n}\left(h_{n}(x)\right)$ and $G_{n}=\left\{x: g_{n}(x)\right.$ $>0\}$. Observe that $\{x: \rho(x, F)>1 / n\} \subset G_{n} \subset O$ and thus $\bigcup_{n=1}^{\infty} G_{n}=O$. Because $X$ is a metric space, $X$ has a $\sigma$-locally finite base $\mathscr{V}^{n=1}=\bigcup_{n=1}^{\infty} \mathscr{V}_{n}$ where each $\mathscr{V}_{n}$ is locally finite and consists of bounded sets. By the above argument, for each $V \in \mathscr{V}$, choose a fixed sequence $\left\{G_{V, k}\right\}_{k} \subset \mathscr{U}_{S}$ such that 
$V=\bigcup_{k=1}^{\infty} G_{V, k}$. Let $\mathscr{G}_{n, k}=\left\{G_{V, k}: V \in \mathscr{V}_{n}\right\}$. Certainly each $\mathscr{G}_{n, k}$ is locally finite. Therefore $\mathscr{G}=\bigcup_{n, k} \mathscr{G}_{n, k} \subset \mathscr{U}_{S}$ is a $\sigma$-locally finite base for $X$.

(b) If, in Lemma 2.6, the dual norm is SC instead of LUC, then the function $g$ constructed there is Gâteaux smooth by Lemma 2.4(b). Moreover, note that $g$ is Fréchet differentiable on finite-dimensional subspaces of $X$ (since distance functions and norms are convex). For $p$ and $g_{1}$ in the proof of Proposition 2.7 (with $g_{1}$ constructed as $g$ is in 2.6), $g_{1} \circ p$ is Gâteaux differentiable everywhere except the origin since for $x_{0}$ and $x_{1}$ fixed, $p\left(x_{0}+t x_{1}\right) \in L$ for all $t$ where $L$ is the linear subspace generated by $x_{0}$ and $x_{1}$. Hence Proposition 2.7 holds with SC replacing LUC in the dual norm and Gâteaux smoothness replacing $C^{1}$-smoothness in the conclusion. To complete the proof of (b) one need only observe that $C^{1}$-smooth functions can now be replaced by Gâteaux differentiable functions in the proof of (a).

Remark 2.8. The full strength of the LUC norm on $X$ was not used in the proof of Lemma 2.6 or elsewhere in the proof of Corollary 2.2. That is, only the strictly weaker condition that every point of $S_{X}$ is strongly exposed was used. This observation yields a result which is, at least formally, better than Theorem 2.2.

\section{Approximation in Hilbert SPACES}

The basic methods of $\S 2$ are used to show that if every norm on a Hilbert space $X$ can be approximated uniformly on bounded sets by functions in $C_{u}^{2}(X)$ (the space of real-valued functions with uniformly continuous second derivative on $X$ ), then the same is true for every uniformly continuous function. In particular, using a proposition of [14] we obtain

Proposition 3.1. There is a norm with Lipschitz derivative on a Hilbert space $X$ that is not a uniform limit on $B_{X}$ of functions in $C_{u}^{2}(X)$.

Two lemmas will be used to prove Proposition 3.1. The first is analogous to Lemma 2.6. In the sequel a function $f$ will be called even if $f(x)=f(-x)$ for all $x \in X$.

Lemma 3.2. Suppose every norm on a Hilbert space $X$ can be approximated uniformly on bounded sets by functions in $C_{u}^{2}(X)$. Then given $\varepsilon>0$ and $a$ uniformly continuous even function $f$ on $B_{X}$, there is a $g \in C_{u}^{2}(X)$ such that $|g(x)-f(x)|<\varepsilon$ for all $x \in S_{X}$.

Proof. Let $\|\cdot\|^{2} \in C_{u}^{2}(X)$ and $\|\cdot\|$ be uniformly convex (the usual Hilbert norm will do). Without loss of generality assume that $f(x) \in[-1,1]$ for all $x \in B_{X}$. Let $\varepsilon>0$ be fixed and choose $N$ so that $7 / 2 N<\varepsilon$. Let

$$
\Delta_{i}=\left[\frac{i-1-N}{N}, \frac{i-N}{N}\right] \text { for } i=1, \ldots, 2 N .
$$

Let $Q_{0}=Q_{2 N+1}=\varnothing$, and for $i=1, \ldots, 2 N$ define

$$
Q_{i}=f^{-1}\left(\Delta_{i}\right) \quad \text { and } \quad \widetilde{Q}_{i}=\overline{\operatorname{conv}}\left(\left(B_{X} \backslash\left(Q_{i-1} \cup Q_{i} \cup Q_{i+1}\right)\right) \cup B_{1 / 2}\right) .
$$

Since $f$ is even, it follows that $\widetilde{Q}_{i}$ is the unit ball of an equivalent norm $\|\cdot\|_{i}$ on $X$. Let $\nu_{i}(x)=\|x\|_{i}$. By uniform convexity and the uniform continuity of $f$ it follows that there exists $\alpha>0$ such that $\nu_{i}(x) \geq 1+\alpha$ for every $x \in Q_{i} \cap S_{X}$ 
and for all $i$. Choose $h_{i} \in C_{u}^{2}(X)$ such that $\left|h_{i}(x)-\nu_{i}(x)\right|<\alpha / 4$ for $x \in B_{3}$. Construct a function $\phi:[0, \infty) \rightarrow[0,1]$ with uniformly continuous second derivative that, moreover, satisfies $\phi(t)=1$ if $t \geq 1+\frac{3}{4} \alpha$ and $\phi(t)=0$ if $t \leq$ $1+\frac{1}{4} \alpha$. Set $r_{i}(x)=\phi\left(h_{i}(x)\right)$. Observe that $\sum_{i=1}^{2 N} r_{i}(x) \geq 1$ for $1 \leq\|x\| \leq 3$. Since $\sum_{i=1}^{2 N} r_{i}(x)$ is uniformly continuous, given $F=\left\{x \in B_{x}: \sum_{i=1}^{2 N} r_{i}(x) \leq\right.$ $\left.\frac{1}{2}\right\}$, the distance from $F$ to $S_{X}$ is $\geq \delta>0$ for some $\delta>0$. The functions $r$ and $\theta$, which are used below, can be expressed as composites of appropriate functions on $\mathbb{R}$ with the norm. Pick $r \in C_{u}^{2}(X)$ such that $r(x)=1$ for all $x \in F$ and $r(x)=0$ for $\|x\| \geq 1$. Let $\alpha_{i}=$ midpoint of $\Delta_{i}$ and for $x \in B_{3}$ define

$$
h(x)=\frac{r(x)+\sum_{i=1}^{2 N} \alpha_{i} r_{i}(x)}{r(x)+\sum_{i=1}^{2 N} r_{i}(x)}
$$

Since $h$ is not necessarily defined on all of $X$, we extend $h$ to a continuous function on $X$. Now construct $g \in C_{u}^{2}(X)$ such that $g(x)=h(x)$ for all $x \in B_{X}$ as follows. Let $g(x)=h(x) \theta(x)$ where $\theta \in C_{u}^{2}(X)$ satisfies $\theta: X \rightarrow$ $[0,1], \theta(x)=1$ for $x \in B_{1}$, and $\theta(x)=0$ for $x \notin B_{2}$. As in the proof of Lemma 2.6, $|g(x)-f(x)|<\varepsilon$ for all $x \in S_{X}$.

Lemma 3.3. Let $X$ be a Hilbert space. If every norm on $X$ can be approximated uniformly on bounded sets by functions in $C_{u}^{2}(X)$, then every real-valued uniformly continuous function can be approximated uniformly on bounded sets by functions in $C_{u}^{2}(X)$.

Proof. The basic proof of Proposition 2.7 works. Let the notation be as in Proposition 2.7 , and let $f$ be uniformly continuous on the bounded subset $F$ of the hyperplane $H$. Note that $Y=X \oplus \mathbb{R}$ is a Hilbert space and that $p_{1}^{-1}(y) \in F$ implies $\Lambda(y) \geq \alpha$ for some fixed $\alpha>0$. Since $\alpha>0$, one can extend $f_{1}$ to a uniformly continuous even function on $B_{Y}$. Apply Lemma 3.2 and proceed as in Proposition 2.7 observing that $p$ is a $C_{u}^{2}$-smooth mapping outside any neighborhood of the origin in $Y$.

Proof of Proposition 3.1. First recall by a duality argument on norms of modulus of convexity of power type 2 , it follows that the norms with Lipschitz derivative are dense among all norms on a Hilbert space. Hence, if every norm with Lipschitz derivative can be approximated uniformly on bounded sets by functions in $C_{u}^{2}(X)$, then so can every norm. Thus by Lemma 3.3 every uniformly continuous function can be approximated uniformly on $B_{X}$ by functions in $C_{u}^{2}(X)$. This contradicts the result of Proposition 3, $\S 7$ in [14] and the remark following it.

Remark. We have been informed by $\mathrm{R}$. Deville (private communication) that he has directly constructed a norm as in Proposition 3.1.

\section{ACKNOWLEDGMENT}

The author wishes to thank the referee for several helpful suggestions, which enhanced the presentation of this note. 


\section{REFERENCES}

1. R. Bonic and J. Frampton, Smooth functions on Banach manifolds, J. Math. Mech. 15 (1966), 877-898.

2. K. Ciesielski and R. Pol, $A$ weakly Lindelöf function space $C(K)$ without any continuous injection into $c_{0}(\Gamma)$, Bull. Polish Acad. Sci. Math. 32 (1984), 681-688.

3. R. Deville, Problèmes de renormages, J. Funct. Anal. 68 (1986), 117-129.

4. R. Deville, G. Godefroy, and V. Zizler, The three space problem for smooth partitions of unity and $C(K)$ spaces, Math. Ann. 288 (1990), 613-625.

5. J. Diestel, Geometry of Banach spaces-selected topics, Lecture Notes in Math., vol. 485, Springer-Verlag, Berlin-New York 1975.

6. M. Fabian, On a dual locally uniformly rotund norm on a dual Vašăk space, Studia Math. (to appear).

7. M. Fabian and S. Troyanski, $A$ Banach space admits a locally uniformly rotund norm if its dual is a Vašák space, Israel J. Math. 69 (1990), 214-224.

8. G. Godefroy, S. Troyanski, J. H. M. Whitfield, and V. Zizler, Smoothness in weakly compactly generated Banach spaces, J. Funct. Anal. 52 (1983), 344-352.

9. $\_$, Locally uniformly rotund renorming and injections into $c_{0}(\Gamma)$, Canad. Math. Bull. 27 (1984), 494-500.

10. R. Haydon, A counterexample to several questions about scattered compact spaces, Bull. London Math. Soc. 22 (1990), 261-268.

11. _ Trees in renorming theory, preprint.

12. R. Haydon and C. A. Rogers, $A$ locally uniformly convex renorming for certain $C(K)$, Mathematika 37 (1990), 1-8.

13. D. McLaughlin, Smooth partitions of unity in preduals of WCG spaces, Math. Z. (to appear).

14. A. S. Nemirovskii and S. M. Semenov, On polynomial approximation of functions on Hilbert space, Mat. Sb. (NS) 21 (1973), 255-277.

15. V. L. Šmulyan, Sur la dérivabilité de la norme dans l'espace de Banach, C. R. (Doklady) Acad. Sci. URSS (N.S.) 27 (1940), 643-648.

16. K. Sundaresan, Geometry and nonlinear analysis in Banach spaces, Pacific J. Math. 102 (1982), 487-498.

17. M. Talagrand, Renormages de quelques $C(K)$, Israel J. Math. 54 (1986), 327-334.

18. H. Torunczyk, Smooth partitions of unity on some non-separable Banach spaces, Studia Math. 46 (1973), 43-51.

Department of Mathematics, University of Alberta, Edmonton, Canada T6G 2G1 